\title{
Adaptation and Cognition of High-Risk Environment Teams in an Input-Mediator-Outcome Framework
}

\author{
Veronika Klára Takács ${ }^{1 *}$, Márta Juhász \\ ${ }^{1}$ Department of Ergonomics and Psychology, Faculty of Economic and Social Sciences, Budapest University of Technology and \\ Economics, H-1521 Budapest, P.O.B. 91, Hungary \\ * Corresponding author, email: takacsveronika@erg.bme.hu
}

Received: 27 October 2016, Accepted: 23 January 2018, Published online: 22 February 2018

\begin{abstract}
The aim of this article is to introduce a conceptual, integrative model of the adaptation of teams in high risk environments. The model is a combination of previous theoretical frameworks of adaptation and task execution, with the aim of providing a comprehensive model for understanding team adaptation specific to high risk environments. We give equal importance to adaptation as an input, a mediator, and an outcome by putting it in an Input-Mediator-Outcome model, although we further wish to emphasize the relevance of team cognition in team adaptation. In addition, we aim to highlight that, depending on the trigger and the already existing characteristics of the team, adaptation might either follow an algorithm-based or a knowledge-based pattern.
\end{abstract}

Keywords

team knowledge, team adaptation, trigger severity, team mental model

\section{Introduction}

\subsection{Definition of teams and team knowledge}

Teams have become an integral part of the daily functioning of most organizations, regardless of the features (e.g. sector, industry, size of the company, etc.) of the organization. Working in teams has many advantages that make it both popular and necessary for successful organizational functioning. As work has become increasingly cognitive and complex, the key factor for today's organizations to gain a competitive advantage is often the knowledge that organizational members possess. However, as tasks are increasingly cognitive and complex, they often exceed an individual's cognitive ability to have complete awareness of all the perspectives of a given task or situation (Cooke et al., 2013). Teamwork is considered to be an effective solution to this contradiction, since working in teams ensures that members can share this cognitive workload and contribute their expertise to different parts of the task (Mathieu et al., 2000; Hámornik and Juhász, 2010), and are thus able to cope with complex situations. In addition, unlike individuals, teams have the ability to quickly and flexibly adapt to unexpected environmental situations, thus increasing organizations' responsivity to changing environment (Soós and Juhász, 2010; Levi, 2011). In this article we use Cooke and her colleagues' definition of a team as "distinguishable sets of two or more people who interact dynamically, interdependently and adaptively toward a common and valued goal, object or mission." (Cooke et al., 2000). As highlighted in this definition, team members are closely interdependent on each other, which indicates that their level of cooperation might have a crucial effect on how successfully they achieve their goal in an environment in which they continuously have to adapt their decisions and behavior to what the current situation requires.

As the phenomenon of teamwork gained relevance in social sciences, more and more research was conducted into the possible factors that contribute to successful teamwork. One crucial factor which was identified is the ability of team members to "be on the same page", which refers to team members' shared knowledge and interpretation of the task, the roles of team members as well as the environment they share. Although the underlying theoretical concept behind fairly similar, this phenomenon is denoted by different terms across different scientific fields (e.g. team mental model, information sharing, transactive memory, group learning, cognitive consensus) (Mohammed and Dumville, 2001). In our study we chose to use the terms 
team mental model and team situational model to name the phenomena which explain how common knowledge between team members helps the team to perform. The concept of team mental model was developed to account for the fluid and implicit coordination in effective teams as well as how teams function in complex, ambiguous situations. Team mental model is defined as team members' shared and organized understanding of knowledge about key elements of teams' relevant environment (Mohammed and Dumville, 2001; Klimoski and Mohammed, 1994; Mohammed et al., 2000). This refers to the collective task- and teamrelevant knowledge that members bring to a situation, and it is acquired by formal training, experience and team discussions. The content of team mental model can include knowledge relevant to teamwork (e.g. the roles and responsibilities of team members). This type of knowledge is called the team-related team mental model. The team mental model can also include knowledge relevant to the task and goal itself (e.g. understanding of the task and strategies), which is called the task-related team mental model. Besides the team mental model, another type of team knowledge is called the team situational model, which is defined as the team's collective understanding of the current situation. It develops in situations while the team is actually engaged in a task and it makes use of the pre-existing team mental model but also incorporates specific characteristics of the current situation. While the team mental model is a long-lasting, more stable form of team knowledge, a team situational model is a more dynamic understanding or knowledge as it changes with the situation (Cannon-Bowers et al., 1993; Cooke et al., 2000; Ellis, 2006).

It is assumed that both the team mental model and the team situational model are critically important factors in teams' performance since they ensure that all the team members possess the same knowledge and interpretation of the situation needed to successfully solve a task or problem. This is especially important in situations that require quick changes in teams' behavior and strategy, sometimes without even having enough time for explicit communication between team members.

\subsection{Team adaptation}

As the nature of tasks has changed in recent years, the application of teams and teamwork has become essential for many organizations. One crucial advantage of team work is the quick and effective adaptation to new or unexpected situations. However, team adaptation has been interpreted in different ways in the literature. To highlight these differences, Maynard and his colleagues (2015) use an Input-Mediator-Outcome (Ilgen et al., 2005) model, where "mediator" - as an alternative to "process"- reflects a broader change of variables that are important mediational influences on variability in team performance. Due to this greater scope for variability the Input-Mediator-Output model is also applied in the present study.

If team adaptation is interpreted as an input, then it refers to the capacity of a team to make needed changes in response to a disruption or trigger (e.g. the training that the team previously received to handle unexpected situations). If team adaptation is defined as a mediator, then it refers to adjustment to relevant team processes in response to a disruption or trigger which would require adaptation. Maynard and his colleagues combine this approach with the team process framework of Marks et al. (2001), and argue that, depending on the type and severity of a problem, teams might engage in an action adaptation process, an "interpersonal" adaptation process or a transition adaptation process. While in an "action" team adaptation process the team members address task-related processes (e.g. medical emergency teams giving the patient the proper treatment), in an "interpersonal" team adaptation they focus on interpersonal processes in the team (e.g. solving an interpersonal conflict between two medical emergency team members), whereas in a "transition" adaptation process they address processes related to their strategy (e.g. medical emergency teams making a new diagnosis and treatment plan after the occurrence of unexpected symptoms). Finally, if team adaptation is defined as an outcome, then it refers to the consequences of the adaptation process, in other words, the impact of the adaptation on outcomes (e.g. medical emergency teams saving a patient's life). In our study we decided to consider these three aspects of adaptation (input, mediator and outcome) as having equivalent roles in a team's functioning, by placing them in an integrative theoretical model.

Adaptation -whether it is defined as input, mediator, or outcome - has special importance for teams working in high risk environments. High risk environments are defined as environments in which there is a more than normal likelihood of damage to one's own health or even loss of life, the health or life of others or to material property (Dietrich and Childress, 2004). Teams working in such environments consist of highly trained individuals, with fairly strict protocols to follow in most scenarios they are envisioned to find themselves in. However, when it comes to non-routine situations, they have to adapt their protocol 
and behaviors to the current situation. These groups are expected to interpret information from multiple systems and to make decisions in non-routine, time-pressured, high-workload situations (Waller, 2004). The adaptation of these teams to abnormal situations is critical, since the way they handle the problem and the decisions they make might have a crucial influence on human lives and/ or the environment. Here we utilize Rasmussen's (1983) theory of human performance to highlight the differences between routine and non-routine situations in terms of task execution. According to Rasmussen, there are two types of tasks, one based on algorithms and the other based on one's knowledge. Algorithm-driven tasks are mostly based on predefined and learned rules and routines while knowledgedriven tasks represent a higher cognitive level where certain cues must be integrated with existing knowledge in order to find a solution to a problem. We argue that teams in high-risk environments perform their tasks mostly within algorithm-based processes, since they are highly trained to follow a strict and detailed protocol during task execution. Adaptation in their case might mean an algorithmic process if they have a protocol to follow in response to the onset of an unexpected situation. However, when a problem occurs for which they do not have rules and routines, adaptation will take the form of a knowledge-based task execution where they need to combine their already existing knowledge with the characteristics of the current situation to eliminate potential danger and to lead the process back to a safer, protocol-based, algorithmic task execution.

\subsection{The aim of the study}

In our study we focus specifically on the adaptation of teams in high-risk environments to non-routine events, from the onset of a problem that necessitates changes until the execution of the given task. To do this we constructed our model (Fig. 1) based on Maynard and colleagues' (2015) approach to team adaptation, by placing it into an input-mediator-outcome framework and by highlighting the importance of trigger severity in adaptation. However, we elected to extend Maynard and colleagues' model with Rasmussen's (1983) model of human performance in order to highlight that the type and severity of the trigger not only entails different focuses in adaptation (e.g. transition, action, interpersonal), but also different task execution (algorithm- or knowledge-based) characteristics as well. We further wish to highlight the importance of team mental models and team situational models in team adaptation, by defining them as mediators in the adaptation process and by matching the different types of these models to the different types of adaptation processes. Finally, we aim to provide an integrative framework of team adaptation by giving equal weight to adaptation as an input, as a mediator and as an outcome, in order to highlight how different -and sometimes fragmented- studies on highrisk environment teams are actually related to each other.

The next section introduces the different elements of our integrative model in detail.

\section{High risk environment teams' adaptation in an i-m-o framework}

\subsection{The definition of trigger}

As highlighted before, one advantage of teamwork over individual problem solving is the capacity of teams to react quickly to unplanned and unexpected changes or disruptions. These changes or disruptions, usually called "triggers", prompt teams to make modifications in order to complete their task (Maynard et al., 2015). Unanticipated changes in the task context may require team members to collectively adapt their roles to the current situation. Lepine (2003) defines these ways of handling situations as reactive (as opposed to anticipated and proactive) in response to a problem, error or discrepancy. They are further characterized by being non-scripted (as opposed to following procedures that are learned in the past) and involve changes in activity and behavior (as opposed to changes in team's knowledge which are not followed by behavioral modification). Triggers can be categorized as either teamwork- or taskwork-focused. While teamwork-based triggers are apt to prompt teams to adjust their interpersonal processes, taskwork-based triggers are apt to prompt teams to adjust their action processes (Maynard et al., 2015). Combining the approach of Lepine and Maynard, we define triggers here as:

Unexpected errors, problems or disruptions in teamwork, for which the team might have no prior knowledge, experience or scripts to solve, and which require immediate changes in either the actions or the interpersonal features of the team, in order to maintain standard performance in task execution.

\subsection{Adaptation as an input}

Team adaptation can be defined as input, in other words, the capacity of a team to make required changes in response to a disruption or trigger. The capacity of a team to change its actions adaptively depends on several factors whose combination can be defined as the actual adaptability of the 
team. These factors can be categorized as individual-level, team-level or organizational level inputs (Maynard, 2015).

On the individual level many studies have sought to explore the individual characteristics of team members that might have an impact on the composition, as well as on the overall performance of the team. Among others, individual inputs may include the cognitive ability of individual team members (Lepine, 2003; Edwards et al., 2006), members' achievement, openness to experience and dependability (LePine, 2003) and members' orientation to learn and perform (LePine, 2005), as well as members' flexibility and adaptability (Maynard et al., 2015).

On the team level inputs are related either to the task or to the team itself. Team-related antecedents include team dynamics, such as conflicts, communication and information transfer in the team; the level of trust within the team, the degree to which team members like or dislike each other as well as the length of time team members have been working together as an actual team. In addition, as Maynard and his colleagues (2015) argue, the level of interdependence within a team might also be an important antecedent of adaptation. In contrast with team-related antecedents, task-related teamlevel inputs might include factors such as decision-making structures (Hollenbeck et al., 2011) and reward systems (Johnson et al., 2006). In addition, potential task-related inputs may be the level to which the team is trained to work together, the number of non-routine situations they are prepared to handle, as well as the knowledge they can bring to bear in non-routine situations.

Typical organizational level inputs include antecedents embedded in the organizational culture, the functioning of the organization and organizational rules and protocols for employees to follow in case of a trigger. For instance, it might be reasonable to assume that in a high-risk environment the prevailing safety culture would be a very strong predictor of how a team will react to an unexpected problem (e.g. the consequences of a false alarm caused by a team reacting to a trigger). Leadership style (e.g. autocratic versus democratic leadership) and hierarchy in the organization might also be relevant factors in determining a team's reaction to a trigger, as may the knowledge management strategy and practices in the organization (e.g. whether the organization is willing and motivated to learn from past events after an error has occurred).

As Maynard et al. (2015) argue, besides individual-, team- and organizational-level antecedents, the severity of the trigger is also a crucial input determining the degree to which a team has to adapt its actions to a particular situation.
Considering inputs from the point of view of a team in a high-risk environment, we argue that the combination of the inputs (individual-, team-, organizational-level antecedents and the severity of the trigger) predetermine whether the team will be able to use already existing protocols and routines to solve a problem or they need to handle the situation on a higher cognitive level by integrating their existing knowledge with the characteristics of the current situation.

\subsection{Adaptation as a mediator}

Adaptation can also be defined as a mediator, in which case it refers to the adjustment of relevant team processes as a response to the trigger that necessitates adaptation. In order to explain the importance of trigger characteristics and severity, Maynard and colleagues (2015) combined their approach with a theory proposed by Marks et al. (2001) on team process, according to which teams engage in three types of processes, and move back and forth between them as the current phase of task accomplishment requires. One of these three processes is called action process and refers to periods of time when teams conduct activities that lead directly to goal accomplishment. Action processes include activities such as monitoring progress toward goals, systems monitoring, and team monitoring and backup responses. When monitoring progress, the team and the system itself is especially relevant for teams working in dynamic environments and relying heavily on machines during their work. The second type of team process is termed an interpersonal process, and refers to processes that teams use to manage interpersonal relationships. These include activities like conflict management, motivating/ confidence building and affect management. We argue that handling interpersonal phenomena like conflict and affect management can have a crucial impact on high-risk environment teams' performance, since rational, levelheaded decisions and implicit communication can only be made if team members have no interpersonal issues hindering task accomplishment. Finally, the third type of process is called a transition process, which involves periods when teams focus primarily on evaluation and planning to guide the accomplishment of their goals. It consists of activities such as mission analysis, goal specification, strategy formulation and planning, and as such it is considered to require the most complex cognitive activity from teams. Given the highly protocolled and rule-based nature of teamwork in high-risk environments, we assume that among the three types of adaptation 
processes, transition has the lowest chance of occurring during teamwork. Although the likelihood of transition activities occurring in high-risk environments is low, the way this type of team process is executed can have a crucial impact on both task accomplishment and human lives and/or the environment.

According to Maynard and colleagues, depending on the severity of the trigger, teams engage in either an action/interpersonal process or a transition process. Trigger severity is defined as the extent to which the change permeates the team, the importance of the trigger and the extent to which it leads to abnormal situations.

\subsubsection{Low/average severity trigger}

We argue that a low or average trigger severity in high-risk environment teams means that, based on the combination of input elements introduced above, they are able to accomplish the task by using the existing protocols and routines.

In such cases teams focus their attention either on action or on interpersonal team processes, depending on the trigger they are facing. If they need to react to a trigger that is interpersonal in nature, they will turn to interpersonal team processes to adjust their characteristics to what the current situation requires. If the trigger is action-related, then the team will engage in action processes that will lead them directly to goal accomplishment (Maynard et al., 2015).

At this point we wish to extend this argument by stating that the severity of the trigger not only leads to different foci in task accomplishment (action, interpersonal, transition focus), but also to different task execution characteristics and different cognitive backgrounds.

Rasmussen's (1983) theory on human performance may, we argue, be applied to highlight the differences that exist between low/average and high trigger severity situations. According to Rasmussen, human performance can be categorized as algorithm- or knowledge-based. Algorithmbased performance derives from pre-learned procedures and algorithms where specific signs trigger stored rules and routines to accomplish the task. Algorithm-based tasks are typically characterized by a relatively low level of freedom. The main requirement in these situations is the correct and rapid execution of the given task.

Based on the theoretical concepts introduced above we argue that in low/average trigger severity situations high-risk environment teams are able to accomplish their task based on previously learned protocols and routines, which means that high-risk environment teams in low/average trigger severity situations actually engage in algorithm-based task execution which then either addresses action- or interpersonal-related issues.

\section{Proposition 1: Low/average severity of trigger in high-risk environment teams means that the team has a suitable combination of individual-, team- and organizational level antecedents to perform an algorithm-based task execution.}

\section{Proposition 2: Algorithm-based task execution in high-risk environment focuses either on action or on interpersonal team processes, depending on the nature of the trigger.}

Since algorithm-based task execution includes the application of pre-learned rules and procedures it requires team members to have the same knowledge and interpretation of the task, procedures and rules. Being on the same page is a very important predictor of team performance, especially for teams that work in high-risk, dynamic environments, since the members often do not have time for explicit communication during task execution. To account for effective implicit coordination between members, team mental models represent collective knowledge about the key elements of the team's environment. They can be interpreted as a shared understanding of aspects related to the task or of the team itself. Given the fact that high-risk environment teams must have a very precise understanding of the task and the functioning of the team in order to follow protocols and routines, we argue that the success of algorithm based taskexecution in high-risk environment teams is mediated by the mental model of the team. Furthermore, since team mental models - depending on their content - can be categorized as being either teamwork- or taskwork-related, we propose that algorithm-based task execution that addresses "action" team processes is mediated by the team's taskwork-related team mental model, while task execution addressing "interpersonal" team processes is mediated by the team's teamwork-related team mental model.

Proposition 3: The success of algorithm-based task-execution (action, interpersonal) in high-risk environment teams is mediated by the team mental model of the team.

Proposition 4: Algorithm-based task execution that addresses "action" team processes is mediated by the team's taskwork-related team mental model. 
Proposition 5: Algorithm-based task execution that addresses "interpersonal" team processes is mediated by the team's teamwork-related team mental model.

\subsubsection{High severity trigger}

In our opinion a high trigger severity in high-risk environment teams leads to a situation where their existing combination of inputs (individual-, team-, organizationallevel antecedents) does not provide enough resources for the team to solve the problem they are faced with based on their already existing knowledge and protocols. As trigger severity increases, the team may need to focus its attention, and efforts to adapt, first on transition processes and only later return to adapting either their action or their interpersonal processes, depending on the nature of the trigger they are facing (Maynard et al., 2015).

It is again worth combining Maynard's approach with that of Rasmussen (1983), who states that besides algorithms, human performance can also have its origin in knowledge-based task execution. Knowledge-driven tasks as opposed to algorithm-based ones- consist of actions on a higher cognitive level, where existing knowledge must be integrated with certain cues of the current situation in order for the team to perform. As such, there is not necessarily a direct relationship between a particular trigger and a specific action in response. While information has to be collected and interpreted before making a decision, there may be more than one correct solution. Teams in this case have relatively more freedom but their cognitive workload is also likely to be higher during knowledge-based task execution.

Based on the combination of the two approaches we argue that as trigger severity increases teams in high risk environments do not have a sufficient combination of inputs to follow already existing protocols and routines and thus high risk environment teams in high trigger severity situations will engage in knowledge-based task execution.

\section{Proposition 6: High level of trigger severity in high-risk environment teams means that the team will engage in transition team process, based on knowledge-driven task execution.}

Since knowledge-based task execution includes such activities as collecting information and integrating existing knowledge with cues of the current situation, it is reasonable to assume that teamwork- and taskworkrelated team mental models per se do not have the capacity to provide a sufficient cognitive background for high-risk environment teams in high trigger severity situations. It may be possible to explain the cognitive background of knowledge-based transition team processes by drawing on the concepts of team situational model and temporal team mental model. Team situational models develop in situations where the team is currently engaged in the task, and as such they represent the team's collective understanding of the specific situation. As this understanding changes with the situation, this is a relatively dynamic mental model which makes use of the pre-existing team mental model but goes further by incorporating the specific characteristics of the current situation. The importance of team situational model is that it guides the team in determining strategies, assessing how the team is proceeding, predicting what team members will do and selecting appropriate actions to take (Cooke et al., 2000) all of which are crucial factors of successful teamwork in situations where an inappropriate interpretation of the current situation might endanger human lives and/or the environment. Although the team situational model is a very significant model of team knowledge which incorporates the team's current understanding of certain situations at a given time, there is also a very specific feature of teams in high-risk environments that the team situational model does not account for: the speed at which they collect information, integrate it with their knowledge and make decision on how to solve the problem. For this reason, we elected to incorporate the theory of "temporal team mental model" in our theoretical background to provide a more comprehensive explanation for team cognition in high trigger severity situations. A temporal team mental model is an "agreement among group members concerning deadlines for task completion, the pacing or speed of activities and the sequencing of tasks". The first component of this definition is "deadlines" which provide cues for delineating time, communicate to members how they should prioritize their time and allow employees to plan and fit their time with the organizational context. The second component is the pacing or speed at which activities take place, while the third component is "the sequencing of tasks" which refers to the agreement between team members on the sequence in which they accomplish the tasks (Mohammed et al., 2015). We argue that high-risk environment teams in high trigger severity situations not only need to have shared team- and taskrelated knowledge, but they also must have a common understanding of the current situation and the time-related 
aspects of the task execution. Therefore, we propose that the success of a knowledge-based transition team process in high-risk environment teams is mediated by the team situational model and the temporal team mental model.

\section{Proposition 7: The success of knowledge-based task execution (transition) in high-risk environment teams is mediated by the team situational model and the temporal team mental model of the team.}

\subsection{Adaptation as an outcome}

If team adaptation is defined as an outcome, then it refers to the consequences of the adaptation process, that is, the impact of adaptation on outcomes. In order to create a theoretical framework that is applicable to any type of high-risk environment team, the outcome - the impact of adaptation on outcomes - in this model is defined on a very general level, namely in terms of performance and collective efficacy.

On this very general level, the performance of high-risk environment teams can be defined as the proper execution of the given task which leads to the achievement of the goal towards which the team was working, resulting in a standard level of performance. In other words, the team is performing on to the level at which it is required to perform, thus meeting the performance criteria related to the task. Applying a general definition of work and career aptitude (Csirszka, 1982), we consider team performance here to be:

\section{Permanent and consistent activity of the team that is performed at least on the average level, without causing harm in one's own health, the health of others, the environment or material properties.}

For high risk environment teams, general performance can involve two possible different scenarios. Either the team is capable of executing the given task and solve the problem it faces by acting exclusively on the basis of algorithms and already existing rules and knowledge (algorithm-based task execution), or the team needs to rebuild its strategy and tasks by taking the requirements of the given situation into account before leading the procedure back to a safer, algorithm-based task execution (knowledge-based task execution).

Outcome cannot, however, only include the successful execution of a given task but it must also consider the psychological consequences of adaptation. A group's expertise does not usually depend only on the raw qualities of the group, but also on how these qualities are perceived by the group members and how it feels to belong to the team. Again, in order to stay on the most general level, collective efficacy is the term used here to describe the psychological outcome of adaptation. Collective efficacy reflects the group's anticipation that it will do well on a particular task (Peterson et al., 2000). While it is widely accepted that a higher level of collective efficacy will result in a high level of task execution, our model also reflects the other direction of this relationship, namely that a high level of task execution will result in an increase in collective efficacy.

Therefore, we propose that in the case of high-risk environment teams this relationship means that a high level of collective efficacy is a result of successful adaptation in response to a trigger.

\section{Proposition 8: A high level of collective efficacy is a general consequence of the adaptation process.}

The level of collective efficacy might even rise when the severity of the trigger is also high. For example, in this case the team has less chance to follow an existing protocol and thus need to employ a more complex, knowledge based task execution with a correspondingly higher degree of challenge to team members. Being able to find a new strategy and then leading the procedure back to an algorithm-based task execution might therefore be perceived as a greater achievement by the team members, which might subsequently be translated into a higher collective efficacy level than in the case of a routine-like, algorithmic task execution.

\section{Proposition 9: A knowledge-based task execution leads to higher collective efficacy than an algorithm- based task execution.}

\subsection{Our model in practice}

In this section we aim to give a comprehensive example from practice to offer a better understanding of our theoretical model. In order to enhance the clarity of the theories combined, we offer a visualization of our model (Fig. 1) that is recommended to read through for a better understanding.

Our theoretical model is here explained through the case of the fire department of a nuclear power plant. Firefighters - just as air traffic controllers in aviation industry or operators in nuclear power plants - are considered as teams working in high risk environments, since there is a more than normal likelihood of damage 


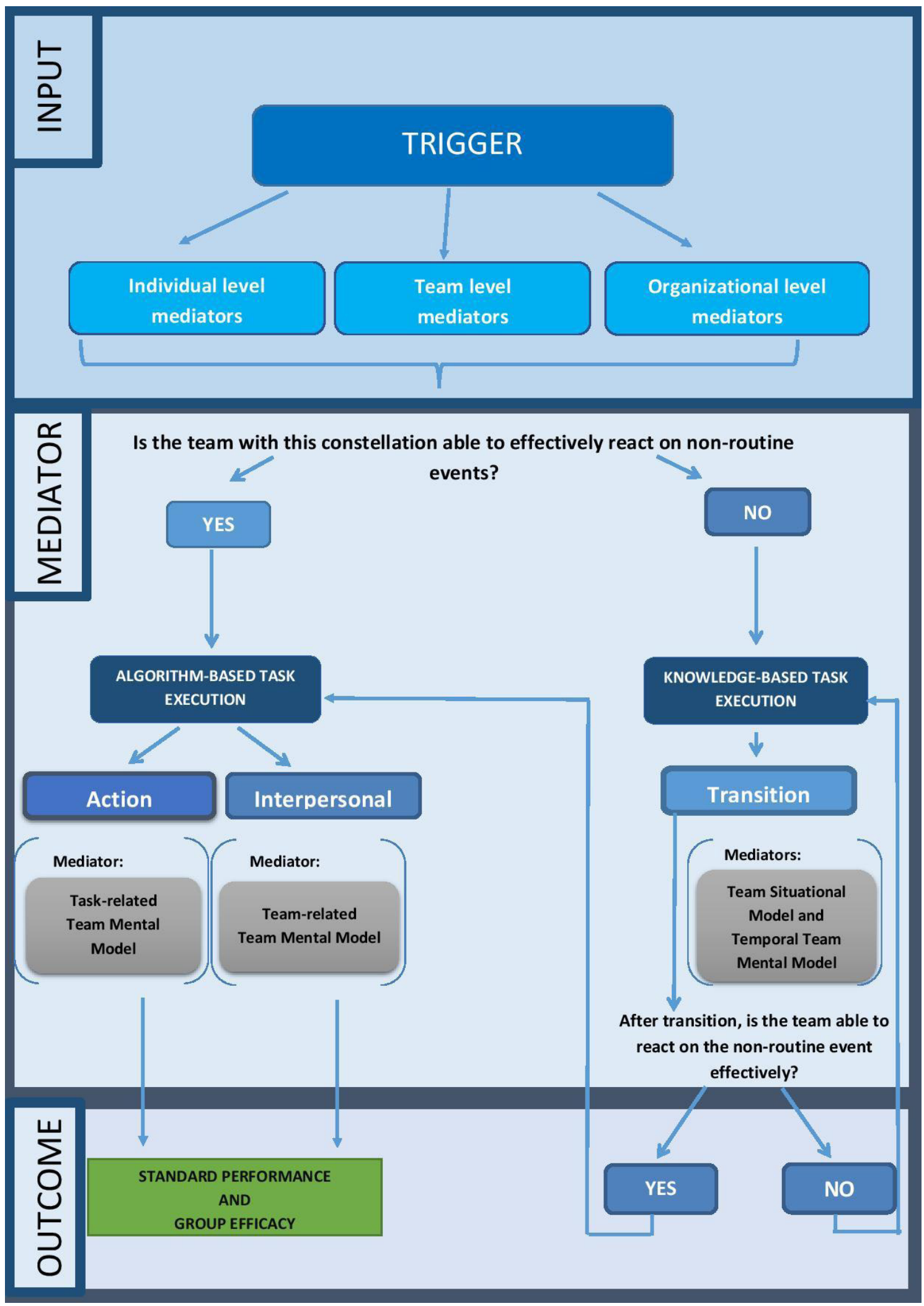

Fig. 1 High risk environment teams' adaptation in an Input-Mediator-Outcome framework 
to their own health or even loss of life, the health or life of others or to material property. Firefighters of nuclear power plants possess very specific knowledge and work according to very strict protocols and rules to follow. The knowledge the team possess can have a serious impact on the outcome of their task.

Adaptation as an input on an individual level in this case applies to the firefighters' professional expertise, their cognitive abilities, their personality traits, level of their adaptability, their organizational tenure. On the team level it applies for instance to the number of months or years for which the fire brigade as a team has been working together, the general atmosphere within the team. On the organizational level it applies for example the prevailing organizational culture (whether it is punitive or rewarding), the leadership style of the head of the fire department, the tools that the department provides for task execution.

If adaptation is viewed as a mediator, then it refers to the adjustment of relevant team processes, in other words, the task execution itself. The following example is an excellent case of a low-severity, algorithm-based situation: the fire department receives an emergency alert according to which there is a fire in the old store of the fire department. There are no dangerous substances or injured individuals reported. After the emergency alert, the fire chief informs the fire brigade who has 2 minutes to leave the department fully dressed and equipped. This case can be considered as a routine task, because they "simply" have to put out the fire for which the brigade has the proper combination of individual-, team-, and organizational level inputs to adapt. Furthermore, there are no additional factors (dangerous substances or injured individuals) that aggravate the task solution. Last but not least, the fact that the fire is in the old store of the fire department further eases the task, since the fire brigade is already familiar with the field. In this case an algorithm-based solution can be executed, for which teamand task-related team mental models serve as the basis for "interpersonal" and "action" adaptational processes. The action adaptation process here is the process of putting the fire out, for which the task-related team mental model is the fundamental knowledge type-knowledge that team members gain during their professional training (e.g. which fire hose is the team supposed to use, how is the team supposed to enter the building and approach the fire, etc.) Interpersonal adaptation process here can be e.g. the way the different sub tasks are partitioned between team members. The basic team knowledge for this adaptation type is the team-related team mental model, which ensures that team members have the same shared knowledge of who does what in the team. If the team's knowledge related to team members' tasks and responsibilities are distorted and unshared, fire fighters might end up in repeatedly and therefore redundantly executing certain sub-tasks (e.g. repeatedly discovering parts of the building that were discovered and considered save previously by other team members). The fire brigade is able to solve the task based on already existing protocols, since they cover routine solutions like this.

The following case is a good representative of a knowledge-based, high trigger-severity scenario: the fire department receives an emergency alert according to which there is a fire in the bunker of the power plant. They further get to know that there is also a person in the building who is probably injured. Furthermore there is a gas tank in the building which is highly explosive. After the emergency alert, the fire chief informs the fire brigade who has 2 minutes to leave the department fully dressed and equipped. This case is obviously more complex than the previous one, since there are additional factors aggravating the task solution: they first must find the injured person and take him out of the bunker as well as they have to find the gas tank before it explodes. They are also a lot less familiar with the field itself. As the fire brigade arrives at the bunker, they realize that the entrance gate is physically blocked, and they have to use specific tools to eliminate it. By entering the building -that has no windows- visibility is even poorer than it normally would be because of the smoke, which makes the exploration of the building extremely difficult. As they find the injured person and take him out, they realize that their fire chief got also seriously injured during the process. It does not only mean that they have one less member in the brigade but it also means that there has to be a new leader of the team. Although the fire brigade has a protocol for this case (the second person in the brigade becomes the fire chief), adapting to this situation and informing the whole team is still a challenge. Last but not least, while they find the gas tank and put the fire out, the entrance of the bunker crashes in, therefore the brigade has to find the emergency exit to leave the building. In this scenario it is obvious that the team has to face a lot of unexpected situations during task solution that requires them to change or modify the strategy they chose to follow (they have to eliminate the blockade of the entrance, they have to solve the situation without the fire chief and adapt to the new one fire chief, and they have to find a different exit from the building). These are what we consider as a "transition" level adaptation, where the fire brigade not only has to possess an 
accurate and shared vision regarding the strategy and the team members' roles (team- and task-related team mental model), but they also have to continuously monitor the environment and make decisions according to these rapidly changing conditions, as well as they have to decide which sub-task they solve at first (referring to team situational model and temporal team mental model, respectively), which is a more complex as well as mentally and physically demanding case. Adaptation here is not only task solution based on protocols, but the constant modification of the strategy -depending on the environmental cues.

As the output for both scenarios -if the adaptation is successful- the collective efficacy of the team will increase, since they were able to solve the situation. However, it is reasonable to hypothesize that the amount of increase will be different for the first and the second scenario. Since the second scenario is a very complex, demanding and fairly dangerous situation, we assume that the collective efficacy of the team will increase more in this case, than in the first one, since it can be considered as a daily, routine event.

\section{Conclusion}

The importance of adaptation in high-risk environments is inevitable, since the way a team reacts to an unexpected error or problem can have a crucial impact on human lives and the environment. However, research on adaptation of highrisk environment teams up to this point has been conducted in several, fragmented fields, and no comprehensive, integrative model has been constructed to date. The present study thus attempts to devise a comprehensive theoretical framework specifically for high-risk environment teams. To build this theoretical model, we chose Maynard and colleagues' meta-analysis of team adaptation, who put the phenomenon in an input-mediator-outcome framework. We highlighted that increases in trigger severity not only indicate different content in terms of the task execution of high-risk environment teams (action, interpersonal or transition process), but it also affects the complexity of cognitive activities. While an algorithm-based task execution with rules and protocols is sufficient for adaptation when the trigger severity is relatively low, as trigger severity increases task execution might need to become knowledgebased in nature, indicating a more complex, higher cognitive level, where knowledge of and specific cues of the situation must be integrated in order to adapt. In our model we also emphasized that, depending on the nature of the problem to be solved, high-risk environment teams might apply different team mental models during the adaptation process.
At a lower level of trigger severity team-related or taskrelated team mental models might serve as the cognitive bases for synchronized team functioning, while as trigger severity increases, task- or team-related team mental models no longer provide a solid and effective cognitive basis for team adaptation. To attempt to explain the cognitive characteristics of teams in high trigger severity situations, the team situational model and the temporal team mental models were introduced as additional necessary cognitive schemata in team adaptation. While a team situational model is necessary for the integration of environmental characteristics in a team's cognitive functioning, the temporal team mental model is included in our model to highlight the importance of a shared understanding among team members of speed, deadlines and sequencing of tasks.

In the comprehensive theoretical model presented here, team adaptation is viewed as an input, a mediator and an outcome. Adaptation as an input refers to the combination of all the individual-, team- and organizational level antecedents on the basis of which the team decides whether or not it has the necessary resources to react to a trigger in an algorithm-based manner. When adaptation is regarded as a mediator, then it refers to all the above mentioned processes of task execution, along with the different team mental models being used during adaptation. Finally, adaptation as an outcome refers to the consequences of the adaptation process. In our theoretical framework, outcomes are defined on a very general level, namely as performance and collective efficacy of the team, in order to create a universal model that is applicable to all HRE teams.

An integrative framework for the adaptation of high-risk environment teams was created with the aim of emphasizing how the fragmented research into team adaptation in various fields are related to each other. On the other hand, recent practices show that the expertise of team members is often over-emphasized compared to other factors that might also have an important impact on the successful functioning of teams in high-risk environments. Using an input-mediator-outcome framework was also important to emphasize how broader organizational and environmental factors also play a role in HRE teams' adaptation. Finally, emphasizing team cognition in the adaptation process was important to highlight that improving team effectiveness can be achieved if the different team mental models that the team is operating with contain accurate and up-todate information and knowledge for successful problem solving. It is therefore crucial that team mental models are kept updated. One of the major sources of improving 
team mental models is learning. Drawing the proper consequences after a problem or error has been responded

\section{References}

Cannon-Bowers, J., Salas, E., Converse, S. (1993) "Shared Mental Models in Expert Team Decision Making." In: Castellan, N. (Ed.). INdividual and Group Decision Making: Current Issues, Hillsdale, NJ, US, Lawrence Erlbaum Associates, Inc., pp. 221-246.

Cooke, N. J., Gorman, J. C., Myers, C. W., Duran, J. L. (2013) "Interactive Team Cognition", Cognitive Science, 37, pp. 255-285. https://doi.org/10.1111/cogs.12009

Cooke, N. J., Salas, E., Cannon-Bowers, J. A., Stout, R. (2000) "Measuring team knowledge," Human Factors, 42, pp. 151-173. https://doi.org/10.1518/001872000779656561

Csirszka, J. (1982) "Munka- és pályaalkalmasság pszichológiája", Tankönyvkiadó, Budapest. Manuscript. (in Hungarian)

Dietrich, R., Childress, T. M. (2004) "Group Interaction in High Risk Environments", Ashgate Pub.

Edwards, B. D., Day, E. A., Arthur, W., Jr., Bell, S. T. (2006) "Relationships Among Team Ability Composition, Team Mental Models, and Team Performance", Journal of Applied Psychology, 91(3), pp. 727-736. https://doi.org/10.1037/0021-9010.91.3.727

Ellis, A. P. J. (2006) "System Breakdown: The Role of Mental Models and Transactive Memory in the Relationship Between Acute Stress and Team Performance", Academy of Management Journal, 49(3), pp. 576-589. https://doi.org/10.2307/20159782

Hámornik, B. P., Juhász, M. (2010) "Knowledge Sharing in Medical Team: Knowledge, Knowledge Management, and Team Knowledge", Periodica Polytechnica Social and Management Sciences, 18(2), pp. 99-107.

https://doi.org/10.3311/pp.so.2010-2.05

Hollenbeck, J. R., Ellis, A. P. J., Humphrey, S. E., Garza, A. S., Ilgen, D. R. (2011) "Asymmetry in structural adaptation: The differential impact of centralizing versus decentralizing team decisionmaking structures", Organizational Behavior and Human Decision Processes, 114, pp. 64-74. https://doi.org/10.1016/j.obhdp.2010.08.003

Ilgen, D. R., Hollenbeck, J. R., Johnson, M., Jundt, D. (2005) "Teams in organizations: From Input-Process-Output models to IMOI models", Annual Review of Psychology, 56, pp. 517-543. https://doi.org/10.1146/annurev.psych.56.091103.070250

Klimoski, R., Mohammed, S. (1994) "Team Mental Model: Construct or Metaphor?", Journal of Management, 20(2), pp. 403-437. https://doi.org/10.1177/014920639402000206

Johnson, M. D., Hollenbeck, J. R., Humphrey, S. E., Ilgen, D. R., Jundt, D., Meyer, C. J. (2006) "Cutthroat cooperation: Asymetrical adaptation to changes in team reward structures", Academy of Management Journal, 49, pp. 103-119. https://doi.org/10.5465/AMJ.2006.20785533

LePine, J. A. (2003) "Team Adaptation and Postchange Performance: Effects of Team Composition in Terms of Members' Cognitive Ability and Personality", Journal of Applied Psychology, 88(1), pp. 27-39. https://doi.org/10.1037/0021-9010.88.1.27 to will result in more accurate team mental models that will finally lead to more effective team adaptation in the future.

LePine, J. A. (2005) "Adaptation of teams in response to unforeseen change: Effects of goal difficulty and team composition in terms of cognitive ability and goal orientation", Journal of Applied Psychology, 90, pp. 1153-1167.

https://doi.org/10.1037/0021-9010.90.6.1153

Levi, D. (2011) "Group Dynamics for Teams", 3rd ed., Los Angeles, CA, Sage.

Marks, M. A., Mathieu, J. E., Zaccaro, S. J. (2001) "A temporally based framework and taxonomy of team processes", Academy of Management Review, 26, pp. 356-376.

https://doi.org/10.2307/259182

Mathieu, J. E., Heffner, T. S., Goodwin, G. F., Salas, E., Cannon-Bowers, J. A. (2000) "The influence of shared mental models on team process and performance", Journal of Applied Psychology, 85, pp. 273-283. https://doi.org/10.4135/9781446286272

Maynard, T. M., Kennedy, D. M., Sommer, S. A. (2015) "Team adaptation: A fifteen-year synthesis (1998-2013) and framework for how this literature needs to "adapt" going forward", European Journal of Work and Organizational Psychology, 24(5), pp. 652-677. https://doi.org/10.1080/1359432X.2014.1001376

Mohammed, S., Klimoski, R., Rentsch, J. (2000) "The Measurement of Team Mental Models: We Have No Shared Schema", Organizational Research Methods, 3(2), pp. 123-165. https://doi.org/10.1177/109442810032001

Mohammed, S., Dumville, B. C. (2001) "Team mental models in a team knowledge framework: expanding theory and measurement across disciplinary boundaries", Journal of Organizational Behavior, 22(2), pp. 89-106. https://doi.org/10.1002/job.86

Mohammed, S., Hamilton, K., Tesler, R., Manusco, V., McNeese, M. (2015) "Time for temporal team mental models: Expanding beyond "what" and "how" to incorporate "when"", European Journal of Work and Organizational Psychology, 24(5), pp. 693-709. https://doi.org/10.1080/1359432X.2015.1024664

Peterson, E., Mitchell, T. R., Thompson, L., Burr, R. (2000) "Collective Efficacy and Aspects of Shared Mental Models as Predictors of Performance over Time in Work Groups", Group Processes \& Intergroup Relations, 3(3), pp. 296-316. https://doi.org/10.1177/1368430200033005

Rasmussen, J. (1983) "Skills, rules, and knowledge; signals, signs, and symbols, and other distinctions in human performance models", IEEE Transacions on Systems, Man, and Cybernetics, 13, pp. 257-266.

https://doi.org/10.1109/TSMC.1983.6313160

Soós, J. K., Juhász, M. (2010) "Capturing team performance differences through communication based analyses of team cognition", Periodica Polytechnica Social and Management Sciences, 18(2), pp. 75-85. https://doi.org/10.3311/pp.so.2010-2.03

Waller, M. J., Gupta, N., Giambatista, R. (2004) "Effects of Adaptive Behaviors and Shared Mental Models on Control Crew Performance", Management Science, 50(11), pp. 1534-1544. https://doi.org/10.1287/mnsc.1040.0210 